\title{
Image distortion in thermoacoustic tomography caused by microwave diffraction
}

\author{
Changhui Li, ${ }^{*}$ Manojit Pramanik, ${ }^{\dagger}$ Geng Ku, ${ }^{\ddagger}$ and Lihong V. Wang ${ }^{\S}$ \\ Biomedical Engineering Department, Washington University in St. Louis, St. Louis, Missouri 63130, USA
}

(Received 18 October 2007; revised manuscript received 11 January 2008; published 28 March 2008)

\begin{abstract}
We report an intrinsic image distortion in microwave-induced thermoacoustic tomography. The distortion, due to microwave diffraction in the object to be imaged, leads to nonuniform excitation of acoustic pressure during microwave illumination. Both numerical simulations and phantom experiments demonstrate this phenomenon. A method of partial correction is also provided.
\end{abstract}

DOI: 10.1103/PhysRevE.77.031923

PACS number(s): 87.57.- s, 42.25.Fx, 42.25.Ja, 42.30.Wb

\section{INTRODUCTION}

Thermoacoustic (TA) tomography (TAT) in biological tissue reconstructs the TA source distribution from the acoustic signals detected over the tissue surface. The TA source is generated from thermal expansion due to a small temperature increase upon absorption of a microwave or radio frequency (rf) pulse (see Fig. 1). TAT combines microwave contrast and ultrasound resolution. Compared with photoacoustic tomography (PAT), TAT has the advantage of imaging deeper into tissue because the microwave attenuation coefficient of soft tissue is smaller than the optical counterpart. TAT has been studied for both laboratory research and clinical applications in breast cancer imaging [1-4].

The acoustic pressure $p$ excited by a microwave pulse can be described by $[5,6]$

$$
\left(\nabla^{2}-\frac{1}{c^{2}} \frac{\partial^{2}}{\partial t^{2}}\right) p(\mathbf{r}, t)=-\frac{\beta}{C_{p}} \frac{\partial H(\mathbf{r}, t)}{\partial t},
$$

where $H(\mathbf{r}, t)$ is a heating function defined as the thermal energy deposited at spatial position $\mathbf{r}$ and time $t$ by the electromagnetic (EM) radiation per unit time per unit volume, $\beta$ is the isobaric volume expansion coefficient in $K^{-1}, C_{p}$ is the specific heat in $J /(K k g)$, and $c$ is the acoustic speed. Here, $\beta$, $C_{p}$, and $c$ are assumed to be uniform constants.

In studying electromagnetic energy absorbed by tissue, the "specific absorption rate" (SAR), which is the EM energy absorbed by a unit mass of tissue per unit time per unit volume, is generally used and can be calculated by

$$
\mathrm{SAR}=\frac{\sigma(\mathbf{r})|\mathbf{E}(\mathbf{r}, t)|^{2}}{\rho(\mathbf{r})}
$$

where $\sigma$ is the conductivity, $\mathbf{E}(\mathbf{r}, t)$ is the real electrical field denoted by a vector

\footnotetext{
*CLI@biomed.wustl.edu

†mano@biomed.wustl.edu

†gku@biomed.wustl.edu

${ }^{\S}$ Corresponding author: Lhwang@biomed.wustl.edu
}

$$
\mathbf{E}(\mathbf{r}, t)=\left(\begin{array}{c}
E_{x}(\mathbf{r}, t) \\
E_{y}(\mathbf{r}, t) \\
E_{z}(\mathbf{r}, t)
\end{array}\right),
$$

$\rho$ is the mass density, and " $|.$.$| " represents the magnitude of$ a vector. The $\sigma|\mathbf{E}|^{2}$ term represents the EM energy absorption. Since $\sigma$ in tissue is generally dispersive, Eq. (2) is exact only for monochromatic source illumination. Because the illumination source in TAT is an EM pulse with a finite bandwidth, we must consider the dispersive effect.

From electrodynamics, the rate of the energy absorption from the EM field per unit volume and per unit time in the tissue can be calculated by the dot product of the current density and electric field. Assuming all absorbed energy generates heat, we can write the generalized formulation of the heating function as

$$
H(\mathbf{r}, t)=\mathbf{j}(\mathbf{r}, t) \cdot \mathbf{E}(\mathbf{r}, t) .
$$

Here $\mathbf{j}$ is the real current density, which is related to the conductivity as

$$
\mathbf{j}(\mathbf{r}, t)=\int_{-\infty}^{+\infty} \hat{\sigma}(\mathbf{r}, \omega) \widetilde{\mathbf{E}}(\mathbf{r}, \omega) e^{-i \omega t} d \omega,
$$

where $\omega$ is the angular frequency of the field and a variable with a tilde denotes its counterpart in the frequency domain. The hat over the conductivity means $\hat{\sigma}$ generally represents a $3 \times 3$ real matrix, which describes the property of an aniso-

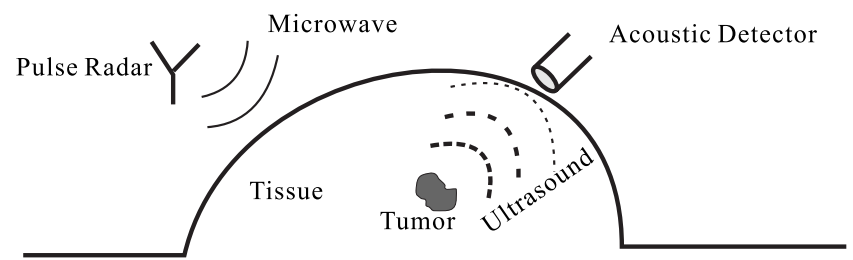

FIG. 1. Illustration of thermoacoustic tomography. A pulsed microwave generated from a radar penetrates the tissue. Because of dielectric contrast, malignant tissue (such as a tumor) usually absorbs more microwave energy and produces a higher temperature increase than the surrounding normal tissue. The temperature rise leads to the generation of ultrasound due to thermoelastic expansion. An acoustic detector receives the ultrasound signals around the tissue, which are used to reconstruct the microwave absorption distribution. 
tropic medium. To simplify the problem, we assume that the tissue is isotropic. Then, the conductivity becomes a real scalar. Therefore, Eq. (4) can be rewritten as

$$
\mathbf{j}(\mathbf{r}, t)=\int_{-\infty}^{+\infty} \sigma(\mathbf{r}, \omega) \tilde{\mathbf{E}}(\mathbf{r}, \omega) e^{-i \omega t} d \omega .
$$

In our experimental system, the microwave source transmits 3.0-GHz center-frequency and $0.5-\mu$ s wide pulses. Since the temporal width of the pulse is $\sim 1000$ times longer than the EM field oscillation period at the center frequency, the source has a narrow bandwidth, and so does the internal field E. We further assume that the conductivity varies slowly within the bandwidth. Thus, we have the approximation

$$
\sigma(\mathbf{r}, \omega) \approx \sigma\left(\mathbf{r}, \omega_{c}\right),
$$

where $\omega_{c}=2 \pi f_{c}$ and $f_{c}=3.0 \mathrm{GHz}$. Therefore, the current density can be approximately written as

$$
\mathbf{j}(\mathbf{r}, t) \approx \sigma\left(\mathbf{r}, \omega_{c}\right) \int_{-\infty}^{+\infty} \widetilde{\mathbf{E}}(\mathbf{r}, \omega) e^{-i \omega t} d \omega=\sigma\left(\mathbf{r}, \omega_{c}\right) \mathbf{E}(\mathbf{r}, t) .
$$

Substituting Eq. (6) into Eq. (3), we obtain

$$
H(\mathbf{r}, t) \approx \sigma\left(\mathbf{r}, \omega_{c}\right) \mathbf{E}(\mathbf{r}, t) \cdot \mathbf{E}(\mathbf{r}, t)=\sigma\left(\mathbf{r}, \omega_{c}\right)|\mathbf{E}(\mathbf{r}, t)|^{2} .
$$

We note that $H$ takes the form of a monochromatic SAR, apart from a factor of $\rho$.

The local electric field $\mathbf{E}(\mathbf{r}, t)$ is determined by the source properties (such as polarization state and illumination direction) as well as the tissue morphology and its microwave dielectric property. Here we assume that the illumination source is a plane wave with linear polarization, which is similar to the source used in our experimental system. From Maxwell's equations, we obtained the linear relation

$$
\widetilde{\mathbf{E}}(\mathbf{r}, \omega)=\hat{\alpha}(\mathbf{r}, \omega) \widetilde{\mathbf{E}}_{i n}(\omega),
$$

where $\widetilde{\mathbf{E}}_{i n}$ is the spectral component of the source plane wave, and $\hat{\alpha}$ is a $3 \times 3$ complex matrix that relates the local field to the source (using a complex matrix recognizes that the local field could have a different polarization state and phase from that of the source field). Let $\hat{n}$ denote the unit vector of the polarization direction, and then we have

$$
\widetilde{\mathbf{E}}_{\text {in }}(\omega)=\widetilde{e}_{\text {in }}(\omega) \hat{n},
$$

where $\widetilde{e}_{\text {in }}(\omega)$ is a scalar representing the amplitude and phase of the source. Then, we obtain the relation in the time domain

$$
\begin{aligned}
\mathbf{E}(\mathbf{r}, t) & =\int_{-\infty}^{+\infty} \hat{\alpha}(\mathbf{r}, \omega) \widetilde{\mathbf{E}}_{\text {in }}(\omega) e^{-i \omega t} d \omega \\
& =\int_{-\infty}^{+\infty} \hat{\alpha}(\mathbf{r}, \omega) \hat{n} \widetilde{e}_{\text {in }}(\omega) e^{-i \omega t} d \omega .
\end{aligned}
$$

As mentioned, we use a narrow bandwidth microwave source in our experimental system. Let the source $e_{\text {in }}(t)$ be the product of a slowly varying function $A(t)$ (envelope) and a high frequency oscillating function (carrier) as

$$
e_{\text {in }}(t)=A(t) \cos \left(\omega_{c} t\right) \text {. }
$$

We can also obtain its spectral component through Fourier transformation as

$$
\widetilde{e}_{\text {in }}(\omega)=\left[\widetilde{A}\left(\omega-\omega_{c}\right)+\widetilde{A}\left(\omega+\omega_{c}\right)\right] / 2 .
$$

Since $A(t)$ is slowly varying, its spectral component $\widetilde{A}\left(\omega^{\prime}\right)$ has significant values only in the narrow bands of $\left|\omega^{\prime}\right| \ll \omega_{c}$.

In practice, the detected acoustic signal usually corresponds to the short-time averaged heating function (7). We thus calculate the short-time average of the intensity of the electric field by

$$
\left\langle|\mathbf{E}(\mathbf{r}, t)|^{2}\right\rangle=\frac{1}{T_{c}} \int_{t}^{t+T_{c}}|\mathbf{E}(\mathbf{r}, t)|^{2} d t,
$$

where $T_{c}=2 \pi / \omega_{c}$. Substituting Eq. (10) and Eq. (12) into Eq. (13) and using the narrow bandwidth approximation, we obtain the following equation after several mathematical manipulations (details in the Appendix)

$$
\left\langle|\mathbf{E}(\mathbf{r}, t)|^{2}\right\rangle \approx \hat{n}^{\top} \alpha^{\dagger}\left(\mathbf{r}, \omega_{c}\right) \hat{\alpha}\left(\mathbf{r}, \omega_{c}\right) \hat{n} A^{2}(t) / 2=\gamma\left(\mathbf{r}, \omega_{c}\right) I_{e}(t),
$$

where $(\cdots)^{\top}$ and $(\cdots)^{\dagger}$ denote the transpose and conjugate transpose, respectively. Further, $I_{e}(t)=A^{2}(t) / 2$ is the electric field intensity of the microwave source, and $\gamma\left(\mathbf{r}, \omega_{c}\right)$ represents the field distribution at the center frequency with source polarization along $\hat{n}$ as

$$
\gamma\left(\mathbf{r}, \omega_{c}\right)=\hat{n}^{\top} \hat{\alpha}^{\dagger}\left(\mathbf{r}, \omega_{c}\right) \hat{\alpha}\left(\mathbf{r}, \omega_{c}\right) \hat{n} .
$$

From Eqs. (7) and (14), we obtain the short-time average of the local heating function

$$
\langle H(\mathbf{r}, t)\rangle \approx \sigma\left(\mathbf{r}, \omega_{c}\right) \gamma\left(\mathbf{r}, \omega_{c}\right) I_{e}(t) .
$$

Let the spatial distribution of the EM energy absorption $s(\mathbf{r})=\sigma\left(\mathbf{r}, \omega_{c}\right) \gamma\left(\mathbf{r}, \omega_{c}\right)$. From Eqs. (8), (9), and (15), we have

$$
s(\mathbf{r})=\frac{\sigma\left(\mathbf{r}, \omega_{c}\right)\left|\widetilde{\mathbf{E}}\left(\mathbf{r}, \omega_{c}\right)\right|^{2}}{\left|\widetilde{e}_{\text {in }}\left(\omega_{c}\right)\right|^{2}} .
$$

Its relation to SAR is

$$
\mathrm{SAR}=\frac{s(r)\left|\widetilde{e}_{\text {in }}\left(\omega_{c}\right)\right|^{2}}{\rho},
$$

where the local total electric field in Eq. (2) is replaced by the local illuminating electric field. The total electric field is the vector sum of the illuminating field and the scattered field.

TAT reconstructs images based on $s(\mathbf{r})$. Previous studies show that large differences exist in conductivity and permittivity between excised malignant and normal breast tissues [7-11]. Thus $\sigma\left(\mathbf{r}, \omega_{c}\right)$ contains information about the position and the shape of a malignant breast tissue object. In previous literature, it was assumed that the reconstructed images represent the real structure of the sample, i.e., the distribution of the dielectric conductivity. This assumption is valid only if $s(\mathbf{r})$ is proportional to the conductivity inside the target (such as a tumor). 
In this paper, we first calculate $s(\mathbf{r})$ by simulating the tissue phantom illuminated by a monochromatic plane wave. Our simulation results point out that $s(\mathbf{r})$ is generally nonuniform, and images generated from TAT are thus distorted even with exact reconstruction methods. Then phantom experimental results are presented to demonstrate both the distortion as well as a correction method. Finally, we discuss the result.

\section{NUMERICAL SIMULATION}

\section{A. Simulation method}

Microwaves with frequencies from several hundred $\mathrm{MHz}$ to several $\mathrm{GHz}$ have wavelengths ranging from meters to centimeters in vacuum. Wavelength in tissue is also comparable with the size of detected sample even with the high tissue permittivity in the microwave range. For instance, a $3.0-\mathrm{GHz}$ microwave has a wavelength of $10 \mathrm{~cm}$ in vacuum and about $4.5 \mathrm{~cm}$ in breast tissue with a relative permittivity of 5, which is comparable with the size of the adult breast. Two diffraction effects must be considered in TAT: first, the global EM field distribution inside the tissue depends on the morphology of the bulk tissue (such as the shape of the breast) as well as the microwave source; second, the local field distribution depends on the local dielectric heterogeneity. Both effects play important roles in TAT since they affect the EM energy dissipation. Although the first effect can be controlled by changing the bulk tissue shape or by controlling the microwave source as is done in pure microwave detection [12], the second effect is difficult to control or predict since the structure of the object (such as a breast tumor) is unknown. In this paper, we focus on the second effect.

Since diffraction is beyond geometric optics, we use the finite-difference time domain (FDTD) $[13,14]$ technique to perform the numerical simulations. The FDTD method, originally developed by Yee [13] in 1966, is suitable for simulating radiative interactions with particles of complex

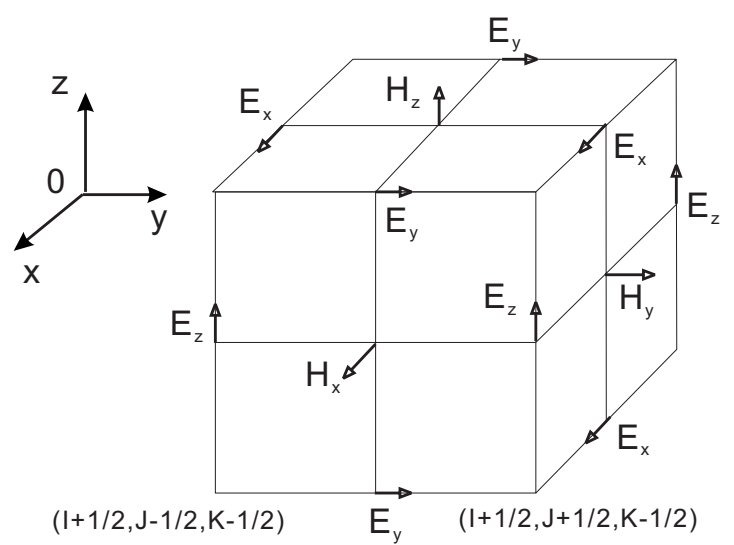

FIG. 2. Three dimensional FDTD grid. $I, J$, and $K$ represent the grid spatial indices. $\Delta x, \Delta y$, and $\Delta z$ are edge length of the grid. Electric fields locate at the center of the edges, and the magnetic fields locate at the center of the faces.

morphology in the diffraction region. The basic principle of this method is to compute the discretized version of the two Maxwell's curl equations

$$
\nabla \times \mathbf{H}=\frac{\partial \mathbf{D}}{\partial t}+\vec{j}, \quad \nabla \times \mathbf{E}=-\frac{\partial \mathbf{B}}{\partial t},
$$

where $\mathbf{E}, \mathbf{D}$ are the electric field and displacement, respectively; H, B are the magnetic field and magnetic flux, respectively; $\vec{j}$ represents the electrical current density, which is equal to $\sigma \mathbf{E}$ in the tissue. The electric and magnetic fields are spatially discretized as shown in Fig. 2.

Then the second order leapfrog algorithm is used to discretize both the spatial and temporal derivatives in Eq. (18). As an example, Eq. (19) gives the time-domain iteration formula for $\mathbf{E}_{x}$ and $\mathbf{H}_{x}$ fields in the vacuum $\left(\vec{j}=0, \mathbf{D}=\varepsilon_{0} \mathbf{E}\right.$, and $\mathbf{B}=\mu_{0} \mathbf{H} ; \varepsilon_{0}$ and $\mu_{0}$ are the vacuum's permittivity and permeability, respectively). To simplify the case, we also choose $\Delta x=\Delta y=\Delta z=\Delta s$.

$$
\begin{aligned}
E_{x}^{n+1}\left(I, J+\frac{1}{2}, K+\frac{1}{2}\right)= & E_{x}^{n}\left(I, J+\frac{1}{2}, K+\frac{1}{2}\right)+\frac{\Delta t}{\varepsilon_{0} \Delta s} \times\left\{H_{z}^{n+1 / 2}\left(I, J+1, K+\frac{1}{2}\right)-H_{z}^{n+1 / 2}\left(I, J, K+\frac{1}{2}\right)+H_{z}^{n+1 / 2}\left(I, J+\frac{1}{2}, K\right)\right. \\
& \left.-H_{z}^{n+1 / 2}\left(I, J+\frac{1}{2}, K+1\right)\right\} H_{x}^{n+1 / 2}\left(I+\frac{1}{2}, J, K\right),=H_{x}^{n-1 / 2}\left(I+\frac{1}{2}, J, K\right)+\frac{\Delta t}{\mu_{0} \Delta s} \times\left\{E_{z}^{n}\left(I+\frac{1}{2}, J-\frac{1}{2}, K\right)\right. \\
& \left.-E_{z}^{n}\left(I+\frac{1}{2}, J+\frac{1}{2}, K\right)+E_{y}^{n}\left(I+\frac{1}{2}, J, K+\frac{1}{2}\right)-E_{y}^{n}\left(I+\frac{1}{2}, J, K-\frac{1}{2}\right)\right\}
\end{aligned}
$$

where $\Delta t$ is the temporal interval, and the superscripts of the fields represent the discretized time-domain indices.

In practice, the computational domain is bounded by proper boundary condition layers. The electric and magnetic fields within the domain are simulated by the finite- difference analog of Maxwell's equations. The presence of a dielectric object in the computational domain is specified by properly assigning permittivity and conductivity values at grid points. In this manner, the FDTD method is flexible in simulating various radiative interactions involving complex 


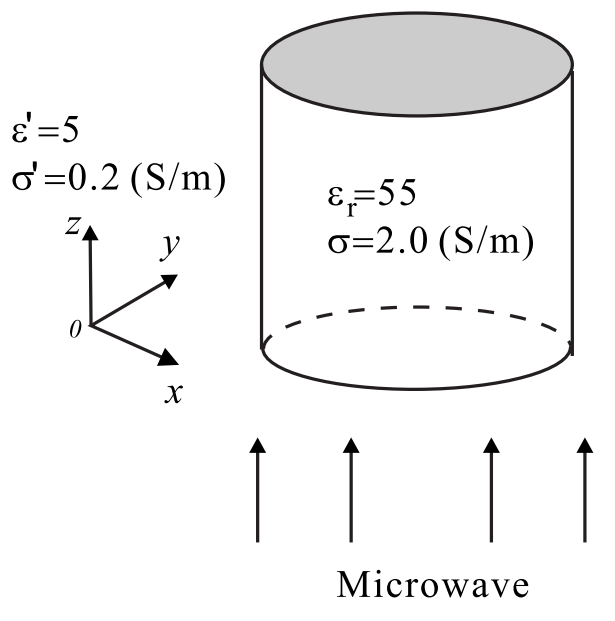

FIG. 3. Simulation model. The malignant tissue is in cylindrical shape, and surrounded by the normal tissue with different dielectric parameters. The microwave illuminates upward along $\hat{z}$ with polarization along $\hat{x}$.

object morphologies and system configurations.

The FDTD code that we developed uses the uniaxial perfect matched layer (UPML) [15] boundary condition to absorb outgoing EM waves. Its accuracy is shown in Ref. [16] by comparing the simulated results of light scattering in vacuum with the analytical results based on the Mie theory. Since tissue, such as breast fat, is also microwave absorbing, we modified the UPML boundary based on the method in Ref. [17] to match the absorbing medium.

In the following subsection, we simulated using TAT to detect a small cancerous tissue inside the breast. The malignant tissue is small enough that the source illuminating microwave in its vicinity can be treated as a plane wave. We further simplify the malignant tissues into cylinders, which is consistent with the experimental phantoms in Sec. III.

\section{B. Simulation results}

The phantoms used in the simulation are dielectrically homogenous cylinders of radii 4.0 and $8.0 \mathrm{~mm}$, with equal lengths of $15.0 \mathrm{~mm}$. Based on the most recent studies $[10,11]$, the two cylindrical tumor phantoms have a relative permittivity and conductivity of

$$
\varepsilon_{r}=55, \quad \sigma=2.0 \mathrm{~S} / \mathrm{m} .
$$

According to Refs. [10,11], the dielectric properties of the surrounding normal breast tissue depend on the per cent of adipose tissue. We chose low water content (more than $85 \%$ adipose tissue) sample values in our simulation, which are about

$$
\varepsilon_{r}^{\prime}=5.0, \quad \sigma^{\prime}=0.2 \mathrm{~S} / \mathrm{m} .
$$

As shown in Fig. 3, a plane wave propagating in the $\hat{z}$ direction with frequency $f=3.0 \mathrm{GHz}$ and $\hat{x}$ polarization is used as the illumination source. A cross section of the FDTD computational region is shown in Fig. 4. The electric field and the magnetic field distributions are calculated by FDTD, and Eq. (16) is then used to obtain $s(\mathbf{r})$.

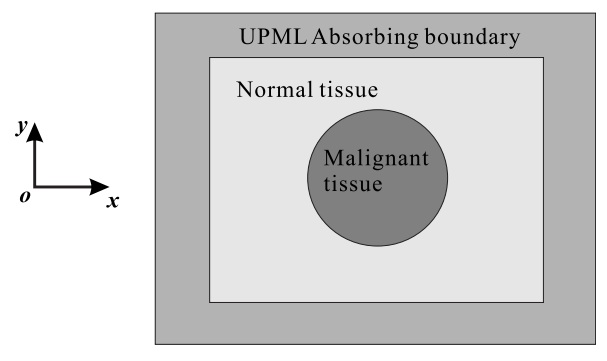

FIG. 4. Computational domain. The EM plane wave is illuminating along the $\hat{z}$ direction, with polarization along $\hat{x}$.

To illustrate the 3D distribution of the EM energy dissipation $s(\mathbf{r})$, we plotted our results on three horizontal cross sections at 1/4,1/2, and 3/4 of the cylinder length (Fig. 5). At each cross section, we calculated the energy absorption both inside and outside the cylinder.

Figure 5(a) shows the simulation result for a cylinder of radius $4.0 \mathrm{~mm}$ and length $15.0 \mathrm{~mm}$, illuminated by a plane microwave along the symmetry axis. It is obvious from the figure that the EM energy dissipation inside the cylinder is nonuniform. The distribution has a "split" pattern with greater $s(\mathbf{r})$ values located near the boundaries intersecting with the $\hat{x}$ direction, which happens to be the source polarization direction. Further, the EM dissipation outside the cylinder is also nonuniform and reaches maximum values that are comparable with the values in the middle part of the cylinder.

Figure 5(b) shows the simulation result for the larger cylinder, of radius $8.0 \mathrm{~mm}$ and length $15.0 \mathrm{~mm}$. The EM energy dissipation inside the cylinder is highly heterogeneous. The EM energy dissipation is "focused" inside the cylinder along the $\hat{z}$ direction, with maximum values located close to the center region, while the EM energy dissipation outside the cylinder is relatively small. Thus the resultant image distortion is different for larger targets.

\section{Image distortions}

The simulation result of the smaller target shown in Fig. 5(a) demonstrates two possible image distortions. The first is "image splitting" along the local polarization direction (it may not be the same as the polarization direction of the illumination source). The image of a single small object could look similar to an aggregation of two (or more) different objects. Another distortion comes from the EM dissipation outside the object. The reconstructed image may have a different shape and a larger size than the actual object.

The image distortion for larger targets is different from that for smaller targets. Since the EM dissipation is focused inside the object, there is little distortion due to energy dissipation outside the target. However, the image distortion for larger targets is not less important, because a highly focused field inside the target causes the image to reveal only parts of the target. Thus, the resultant image distortion includes shrinkage in size and artificial internal structures.

In addition to the distortion in the morphology reconstruction, the values of $s(\mathbf{r})$ are also very different in the two 


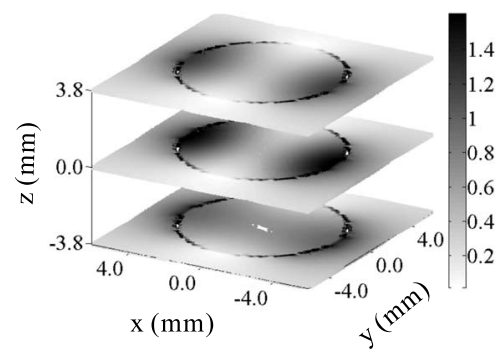

(a)

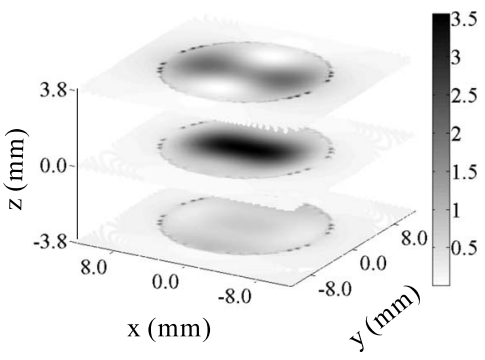

(b)
FIG. 5. Simulation results of $s(\mathbf{r})$ for homogenous cylinders with a length of $15.0 \mathrm{~mm}$ each. (a) For the cylinder with a radius of $4.0 \mathrm{~mm}$. (b) For the cylinder with a radius of $8.0 \mathrm{~mm}$. simulation models, although they have the same conductivity. This difference will affect the quantitative analysis of the reconstructed images.

The size of the target here is relative to the illuminating wavelength. For instance, if we use a shorter wavelength, such as half of the one we used in our experiment, the cylinder with radius $4 \mathrm{~mm}$ will be considered as a "large" target, and the corresponding image distortion pattern will be different.

\section{EXPERIMENT}

Experiments were carried out to demonstrate the image distortion in TAT. Figure 6 shows two phantoms made of porcine fat. Each phantom had a hole in the middle filled with a water-based gel sample. The gel and the porcine fat were used to mimic tumor and normal breast tissue, respectively. The gel has much higher refractive index and conductivity than the fat, and thus the phantom qualitatively simulate the differences in dielectric properties of a breast tumor and normal breast tissue. Both samples are made of $8 \%$ gelatin and $92 \%$ water. The gel in the sample was in the form of cylinders of length $15 \mathrm{~mm}$, with radii of $4 \mathrm{~mm}$ and $8 \mathrm{~mm}$, respectively. The samples were placed inside a bath of mineral oil, which has a very small absorption coefficient for microwaves and provides good acoustic coupling.

A $3.0-\mathrm{GHz} 0.5-\mu$ s microwave pulse source was used in the experiment. The source included a commercial magnetron (Marconi, MG5223) and home-made circuit. It had a repetition rate of $10 \mathrm{~Hz}$ to irradiate the sample using an airfilled pyramidal horn type antenna (WR284 horn antenna W/EEV flange, HNL Inc.) with an opening of 7.34 $\times 10.69 \mathrm{~cm}^{2}$ and $T E_{10}$ mode. The pulse energy was estimated to be around $10 \mathrm{~mJ}$. For detecting the ultrasound sig-

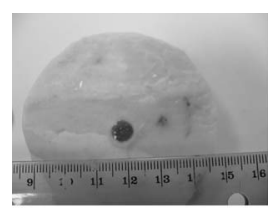

(a)

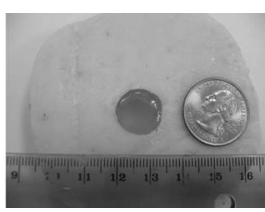

(b)
FIG. 6. Porcine fat phantoms used in the experiment. (a) Phantom with a small hole (radius of $4 \mathrm{~mm}$ ) in the middle filled with gel. (b) Phantom with a large hole (radius of $8 \mathrm{~mm}$ ) in the middle filled with gel. The microwave illuminates upward along $\hat{z}$, with polarization along $\hat{x}$. nal, we used a $2.25-\mathrm{MHz}$ central frequency, $1.27-\mathrm{cm}$ diameter active area unfocused transducer (ISS $2.25 \times 0.50 \mathrm{NF}$, Krautkramer). The transducer was scanned around the sample on a plane perpendicular to the gel cylinder axis for a full $360^{\circ}$, with 160 stops, as shown in Fig. 7. At each stop, the signal was first amplified by a low-noise pulse amplifier (5072PR, OlympusNDT), then filtered electronically and finally recorded using an oscilloscope (TDS640A, Tektronix). The signal was recorded 100 times at each stop, then averaged to reduce noise and transferred to a PC for image reconstruction. A function generator $(10 \mathrm{MHz}$ pulse generator 4010, Global Specialties) triggered the microwave pulses and synchronized the sampling of the oscilloscope. A simple delay-and-sum reconstruction algorithm [18] was used to reconstruct the 2D images from the acquired data (shown in Fig. 8). Because the thickness of the image slice is $\sim 12.7 \mathrm{~mm}$ (about the diameter of the transducer's active area), the images correspond to the overlap of the patterns of different layers in Fig. 5.

Significant distortions are shown in Fig. 8 when the image is compared to the actual phantom sample (Fig. 6). Reconstructed image of the target with smaller radius [Fig. 8(a)] clearly shows the "splitting" phenomenon. Two dark regions are separated approximately along $\hat{x}$, the polarization direction of the microwave source. We can also find gray regions outside the dark area along $\hat{x}$, which means that there are signals from the surrounding medium as expected from the simulation. These distortions are consistent with the numerical simulation.

The reconstructed image for the larger target shows no "splitting" but a more focused pattern, as shown in Fig. 8(d).

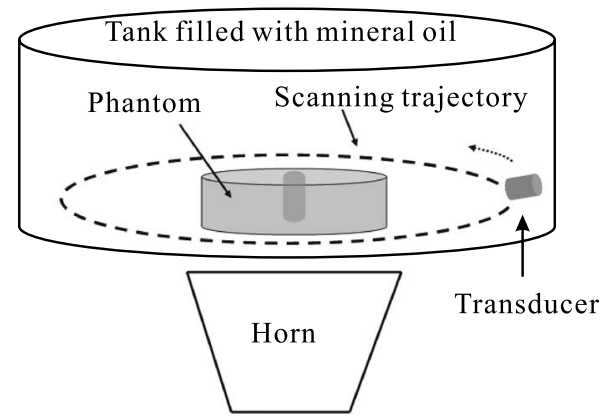

FIG. 7. Experiment setup. The phantom and transducer are placed in a tank filled with the mineral oil. Microwave is illuminating upward from the horn. The dashed line represents the scanning trajectory. 

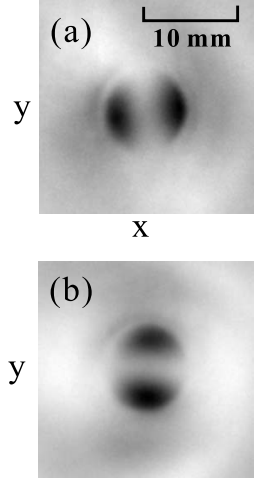

$\mathbf{X}$

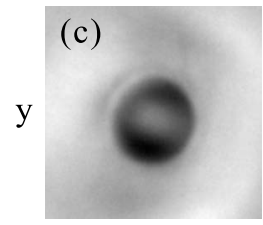

$\mathbf{X}$

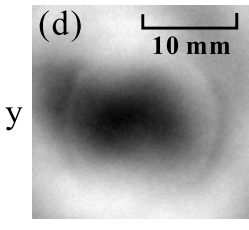

$\mathbf{X}$

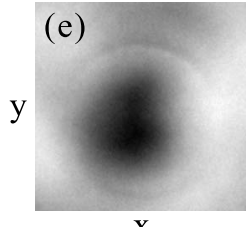

$\mathbf{X}$

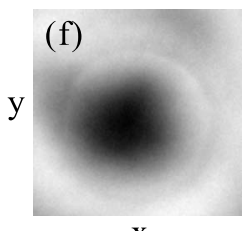

$\mathrm{X}$
FIG. 8. Reconstructed images by delay-and-sum reconstruction algorithm. (a) Image of the phantom with a water gel cylinder of radius $4.0 \mathrm{~mm}$, using the illumination source with $\hat{x}$ polarization. There is a clear "split" pattern. (b) Image of the phantom with a water gel cylinder of radius $4.0 \mathrm{~mm}$, using the illumination source with $\hat{y}$ polarization. (c) Image of the average of (a) and (b). (d) Image of the phantom with a water gel cylinder of radius $8.0 \mathrm{~mm}$, using the illumination source with $\hat{x}$ polarization. (e) Image of the phantom with a water gel cylinder of radius $8.0 \mathrm{~mm}$, using the illumination source with $\hat{y}$ polarization. (f) Image of the average of (d) and (e).

A dominant but shrunken dark region is located close to the center, as expected from the simulation. There is also a gray region close to the left boundary, which is not expected from the simulation.

Although the image distortion in this experiment can be explained by the numerical simulation, not all distortion patterns shown in the simulation are found here, due to differences between the theoretical simulations and the actual experiments. For instance, the permittivity and conductivity values used in the simulation are those of human breast fat, which may not be the same as those of porcine fat; also the background medium (porcine fat) itself is not homogenous; the water based gel contains some small air bubbles, and the microwave may not be aligned to propagate in the exact direction of the cylinder axis. All these discrepancies could change the EM energy dissipation from the theoretical prediction.

We also rotated the polarization direction of the microwave source to get other images, as shown in Figs. 8(b) and $8(\mathrm{e})$. Then we averaged the reconstructed images from the two perpendicular polarization states. The results are shown in Figs. 8(c) and 8(f) for the smaller and larger targets, respectively. From Fig. 8(c), although there is still distortion in the center region, the image no longer looks like split into two parts. As expected, this solution does not reduce the image distortion for the larger target [Fig. 8(f)].

\section{CONCLUSIONS AND DISCUSSION}

In this paper, we reported an image distortion mechanism in TAT. Both numerical simulations and experiments demonstrated this phenomenon. The distortion arises from the diffraction effects of the microwave interacting with the tissue. The distortion not only depends on the dielectric properties of the tissue (normal and malignant) and the microwave source, but also on the geometry (location, size, orientation, etc.) of the target. In reality, tumors may have various morphologies. Thus the distortion patterns will not be limited to the patterns shown in this paper.

New algorithms are needed to recover the real target morphology from the distorted image. Experimentally, the distortion for small targets can be partially alleviated by rotating the polarized EM source. Another possible solution to the image "splitting" in smaller targets is to use a circularly polarized EM wave as the illumination source. Although we used a monochromatic source approximation in this paper, the discussions in the introduction can be straightforwardly generalized to study wide band illuminations.

\section{ACKNOWLEDGMENT}

This project was sponsored in part by the National Institute of Health Grants No. R01 NS46214 and R01 EB000712.

\section{APPENDIX: DERIVATION OF EQ. (14)}

From Eq. (10), we have

$$
\begin{aligned}
|\mathbf{E}(\mathbf{r}, t)|^{2}= & \iint_{-\infty}^{+\infty}\left[\hat{\alpha}(\mathbf{r}, \omega) \hat{n} \widetilde{e}_{\text {in }}(\omega)\right] \\
& \times\left[\hat{\alpha}\left(\mathbf{r}, \omega^{\prime}\right) \hat{n} \widetilde{e}_{\text {in }}\left(\omega^{\prime}\right)\right]^{\dagger} e^{-i\left(\omega-\omega^{\prime}\right) t} d \omega d \omega^{\prime} \\
= & \iint_{-\infty}^{+\infty} \hat{n}^{\top} \alpha^{\dagger}\left(\mathbf{r}, \omega^{\prime}\right) \hat{\alpha}(\mathbf{r}, \omega) \hat{n} \\
& \times \widetilde{e}_{\text {in }}(\omega) e_{\text {in }}^{*}\left(\omega^{\prime}\right) e^{-i\left(\omega-\omega^{\prime}\right) t} d \omega d \omega^{\prime},
\end{aligned}
$$

where $(\cdots)^{\top}$ and $(\cdots)^{\dagger}$ denote the transpose and conjugate transpose, respectively. From Eq. (9), we have

$$
\begin{aligned}
\tilde{e}_{\text {in }}(\omega) e_{\text {in }}^{*}\left(\omega^{\prime}\right)= & \frac{1}{4}\left[\tilde{A}^{*}\left(\omega^{\prime}-\omega_{c}\right)+\tilde{A}^{*}\left(\omega^{\prime}+\omega_{c}\right)\right] \\
& \times\left[\tilde{A}\left(\omega-\omega_{c}\right)+\tilde{A}\left(\omega+\omega_{c}\right)\right], \\
& =\frac{1}{4}\left[\tilde{A}^{*}\left(\omega^{\prime}-\omega_{c}\right) \tilde{A}\left(\omega-\omega_{c}\right)+\tilde{A}^{*}\left(\omega^{\prime}+\omega_{c}\right) \tilde{A}(\omega\right. \\
& \left.+\omega_{c}\right)+\widetilde{A}^{*}\left(\omega^{\prime}-\omega_{c}\right) \tilde{A}\left(\omega+\omega_{c}\right) \\
& \left.+\widetilde{A}^{*}\left(\omega^{\prime}+\omega_{c}\right) \tilde{A}\left(\omega-\omega_{c}\right)\right] .
\end{aligned}
$$

According to the narrow bandwidth approximation, $A(\omega)$ has significant values only when $|\omega| \ll \omega_{c}$. Then substituting Eq. (A2) into Eq. (A1), we have 


$$
\begin{aligned}
|\mathbf{E}(\mathbf{r}, t)|^{2} \approx & \hat{n}^{\top} \alpha^{\dagger}\left(\mathbf{r}, \omega_{c}\right) \hat{\alpha}\left(\mathbf{r}, \omega_{c}\right) \hat{n} A^{2}(t) / 2 \\
& +\hat{n}^{\top} \alpha^{\dagger}\left(\mathbf{r}, \omega_{c}\right) \hat{\alpha}\left(\mathbf{r},-\omega_{c}\right) \hat{n} \\
& \times \iint_{-\infty}^{+\infty} \widetilde{A}^{*}\left(\omega^{\prime}-\omega_{c}\right) \tilde{A}\left(\omega+\omega_{c}\right) e^{-i\left(\omega-\omega^{\prime}\right) t} d \omega d \omega^{\prime} \\
& +\hat{n}^{\top} \alpha^{\dagger}\left(\mathbf{r},-\omega_{c}\right) \hat{\alpha}\left(\mathbf{r}, \omega_{c}\right) \hat{n} \\
& \times \iint_{-\infty}^{+\infty} \widetilde{A}^{*}\left(\omega^{\prime}+\omega_{c}\right) \tilde{A}\left(\omega-\omega_{c}\right) e^{-i\left(\omega-\omega^{\prime}\right) t} d \omega d \omega^{\prime},
\end{aligned}
$$

where we use the relation $\hat{\alpha}(r,-\omega)=\hat{\alpha}^{*}(r, \omega)$ from Eq. (8).
The narrow bandwidth approximation also determines that the two integral terms have significant values only when $\mid \omega$ $-\omega^{\prime} \mid \approx 2 \omega_{c}$. Thus, the short-time average of the exponential terms in Eq. (A3) will be

$$
\begin{aligned}
\int_{t}^{t+T_{c}} e^{-i\left(\omega-\omega^{\prime}\right) t} d t & \approx \frac{e^{-i\left(\omega-\omega^{\prime}\right) t}}{ \pm 2 i \omega_{c}}\left(e^{-i\left(\omega-\omega^{\prime}\right) T_{c}}-1\right) \\
& \approx \frac{e^{-i\left(\omega-\omega^{\prime}\right) t}}{ \pm 2 i \omega_{c}}\left(e^{ \pm 2 i \omega_{c} T_{c}}-1\right)=0 .
\end{aligned}
$$

Finally, the short-time average of $|\mathbf{E}(\mathbf{r}, t)|^{2}$ is proved to be Eq. (14).
[1] R. A. Kruger, K. K. Kopecky, A. M. Aisen, D. R. Reinecke, G. A. Kruger, and W. L. Kiser, Radiology 221, 275 (1999).

[2] R. A. Kruger, W. L. Kiser, D. R. Reinecke, G. A. Kruger, and R. L Eisenhart, in Microwave Symposium Digest, 1999 IEEE MTT-S International, edited by W. L. Kiser (IEEE, 1999) Vol. 2, pp. $591-594$

[3] L. Wang, X. Zho, H. Sun, and G. Ku, Rev. Sci. Instrum. 70, 3744 (1999).

[4] G. Ku and L. V. Wang, Med. Phys. 27, 1195 (2000).

[5] V. E. Gusev and A. A. Karabutov, Laser Optoacoustics (AIP, New York, 1993).

[6] G. J. Diebold, T. Sun, and M. I. Khan, Phys. Rev. Lett. 67, 3384 (1991).

[7] S. Chaudhary, R. Mishra, A. Swarup, and J. Thomas, Ind. J. Biochem. Biophys. 21, 76 (1984).

[8] A. J. Surowiec, S. S. Stuchly, J. R. Barr, and A. Swarup, IEEE Trans. Biomed. Eng. 35, 257 (1988).

[9] W. T. Joines, Y. Zhang, C. X. Li, and R. L. Jirtle, Med. Phys.
21, 547 (1994).

[10] M. Lazebnik et al., Phys. Med. Biol. 52, 6093 (2007).

[11] M. Lazebnik et al., Phys. Med. Biol. 52, 2637 (2007).

[12] S. Semenov, R. Svenson, A. Boulyshev, A. Souvorov, V. Borisov, Y. Sizov, A. Starostin, K. Dezern, G. Tatsis, and V. Baranov, IEEE Trans. Biomed. Eng. 43, 869 (1996).

[13] K. S. Yee, IEEE Trans. Antennas Propag. AP-14, 302 (1966).

[14] A. Taflove and S. Hagness, Computational Electrodynamics: The Finite-Difference Time-Domain Method (Artech, Boston, MA, 2000).

[15] Z. S. Sacks, D. M. Kingsland, R. Lee, and J. F. Lee, IEEE Trans. Antennas Propag. 43, 1460 (1995).

[16] C. Li, G. W. Kattawar, and P. Yang, J. Quant. Spectrosc. Radiat. Transf. 89, 123 (2004).

[17] W. Sun, N. G. Loeb, and Q. Fu, Appl. Opt. 41, 5728 (2002).

[18] C. G. A. Hoelen, F. F. M. de Mul, R. Pongers, and A. Dekker, Opt. Lett. 23, 648 (1998). 